\title{
High-speed video capture of anterior cruciate ligament tearing in human and porcine ex vivo specimens
}

\author{
Smith DT ${ }^{1 *}$, Cook SL ${ }^{1 *}$, Gillespie MA ${ }^{1,2}$, Stevens $\mathrm{KA}^{3}$, Reynolds $\mathrm{HC}^{4}$, Hurd RC ${ }^{5}$ and Wisco JJ", \\ ${ }^{1}$ Department of Physiology and Developmental Biology, Neuroscience Center, Brigham Young University, Provo, USA \\ ${ }^{2}$ University of Texas Southwestern Medical Center, Dallas, Texas, USA \\ ${ }^{3}$ Department of Mechanical Engineering, Brigham Young University, Provo, USA \\ ${ }^{4}$ Department of Neurobiology and Anatomy, University of Utah School of Medicine, Salt Lake City, USA \\ ${ }^{5}$ Department of Mechanical and Aerospace Engineering, Incompressible Fluids Lab, Utah State University, Logan, USA \\ \#Authors contributed equally to the work
}

\begin{abstract}
The tearing of ligaments during sports activities is one of the most common injuries players sustain. Of these ligament tears, the Anterior Cruciate Ligament (ACL) is one of the most prevalent. Although the anteromedial (AM) and posterolateral (PL) bundles of the ACL have been well described, biomechanics research focuses primarily on the strength of the ligament under tension forces. And yet, post-reconstruction tears remain prevalent. To date, no one has captured the tearing of the ACL using high-speed video. We hypothesize that high-speed filming of an artificially induced tear of the human and porcine ACL will reveal the organization of the ligament fibers and provide insight on the ligament's structural organization and integrity. We filmed scalpel-induced tears of three human and three porcine ACL's using a high-speed (6000 fps) camera. With the femur fixed securely on a table surface, and the tibia hanging over the edge, resulting in a flexed knee joint at 90 degrees, a $2.2 \mathrm{~kg}$ weight was attached to the tibia in order to induce tension on the ACL. The ACL was then cut anteriorly to a depth of approximately 1-3 $\mathrm{mm}$ with a scalpel, while a high-speed video camera recorded the unraveling of the anteromedial (AM) and posterolateral (PL) bundle fibers. At the initiation of the human ACL tears, there was a consistent, subtle release of tension visible as a ripple in the most anterior fibers of the AM bundle. Furthermore, the tear pattern revealed that the PL bundle acts as a posterior post, with the AM bundle wrapping tightly around it. In contrast, the porcine bundles were arranged distinctly from one another, with a noticeable, but slight gap of 1-2 mm: Unlike the human ACL, the porcine ACL bundles tore independently. High-speed video of artificially induced ACL tears reveals a distinct difference in the structural arrangement of the AM and PL bundles between human and porcine samples. The independent tearing of the porcine AM and PL bundles suggests that human ACL reconstruction integrity could benefit from a similar two-bundle arrangement. High-speed video of artificially torn human and porcine ACL's reveal an important difference in the geometric arrangement of the two bundles between the two species: Namely, human ACL bundles are tightly wound, but porcine ACL bundles are arranged as seemingly separate ligaments that tear independently. The video footage suggests that human ACL reconstruction may benefit from a distinct two-bundle geometry.
\end{abstract}

\section{Introduction}

The tearing of the Anterior Cruciate Ligament (ACL) is among one of the most common sports injuries. More than 80,000 documented cases of ACL tearing occurs with a $15 x$ greater possibility of re-tearing after reconstruction $[1,2]$. The ACL is known to be comprised of two fiber bundles, namely the anteromedial (AM) and posterolateral (PL) bundles $[3,4]$. ACL biomechanics research has primarily focused on forces on the strength of the ligament. Investigators have shown that a 20-degree flexion of the knee and internal (but not external) tibial torsion will result in increased strain on the ACL, sufficient to produce tensile and shear loads within the ACL that may result in tearing [4-7].

Animal models for the human ACL have included cow, goat, sheep, dog and pig [8]. The porcine ACL has routinely been used as an animal model of ex vivo tear dynamics. These studies however, have stated that although the animal models present valuable similarities to the human ACL, they do also present key differences in length, size and structure. To date, little has been done to visualize the dynamics of the ligament tear itself in either animal models or humans.

The purpose of this project was to visually record artificial grade 3 ACL tearing in human cadaveric specimens and qualitatively compare the dynamics to that of porcine tears. The focus was on both the AM and PL fiber bundles as they unravelled during the event of the tear. The unique nature of this project was the use of high-speed video footage to capture the ligament as it underwent a tear. The footage was then transferred to a visual platform where a researcher could more easily view the unravelling bundles. This dynamic footage gave us greater insight as to the mechanics and structure of the ACL, while in motion of a tear, allowing deeper understanding as to the geometrical arrangement of human v. porcine fiber bundles.

Correspondence to: Jonathan J Wisco, Ph.D., Associate Professor, Brigham Young University, Department of Physiology and Developmental Biology Neuroscience Center Laboratory for Translational Anatomy of Degenerative Diseases and Developmental Disorders (TAD4) 4005 LSB Provo, Utah 84602-5255, USA, Tel: 310-746-6647; 801-422-2402; Fax: 801-422-0004; E-Mail: jjwisco@byu.edu

Key words: ACL tear, high-speed filming, anteromedial fiber bundle, posterolateral fiber bundle

Received: February 17, 2018; Accepted: February 26, 2018; Published: March 03,2018 
Previous studies have primarily set their focus on relative strengths and tear dynamics of the AM and PL bundles [4-7]. In this pilot study, a greater emphasis was placed on documenting, using high-speed filming, the anatomy of the two bundles as they unravelled under weighted tension after being cut with a scalpel. Additionally, it is noted that in previous research the porcine ACL was routinely used as a substitute model for the Human ACL, while recognizing that it contained key differences [8]. However, this study contrasts the geometrical arrangement of the AL and PM bundles between species, and serves as a platform to suggest, based on the tear dynamics we observed, that ACL reconstructive surgery outcomes may improve by using grafts that are built akin to the porcine geometry.

\section{Methods}

In this IRB exempt study utilizing non-human tissue, we obtained three unembalmed opportunity specimen human cadaveric (from University of Utah School of Medicine Body Donor program) and porcine knees (from Gary's Meat, Payson, UT; and Circle V Meat Co., Spanish Fork, UT), then dissected them to expose the ACL. To begin, we made a transverse cut two-thirds down the tibial shaft distally to remove the foot and ankle. Next, an extensive dissection of all musculature and surrounding connective tissue of the upper and lower leg was performed to completely expose the appendicular bones. The fibula was then removed from the attachment site of the tibia. Intricate dissection of knee tissues was then done to reveal the ACL by removing quadriceps tendon, patellar ligament, medial collateral ligament, lateral collateral ligament, posterior cruciate ligament, and tibial menisci. A 45-degree angle cut from anterior superior to posterior inferior was then made through the epicondyles of the distal femoral head to better reveal the ACL for filming. In order to increase visibility of ligament fibers, we used a stain comprised of powdered sugar and blue food coloring dye (9) that revealed fine fiber bundle architecture (Figure 1). The femur was secured to a horizontal platform with two screws and bolts located towards the proximal head of the femur to avoid the bone from splitting when screws were placed (Figure 2).

The first human ACL tear was induced with an anterior cut on the proximal third of the AM bundle. The introduction of a cut on the AM bundle was not made with the intent to expressly mimic natural ACL failure. Rather, it was to manually initiate the tearing of the fiber bundles in order to record the ACL failure in a specific time frame

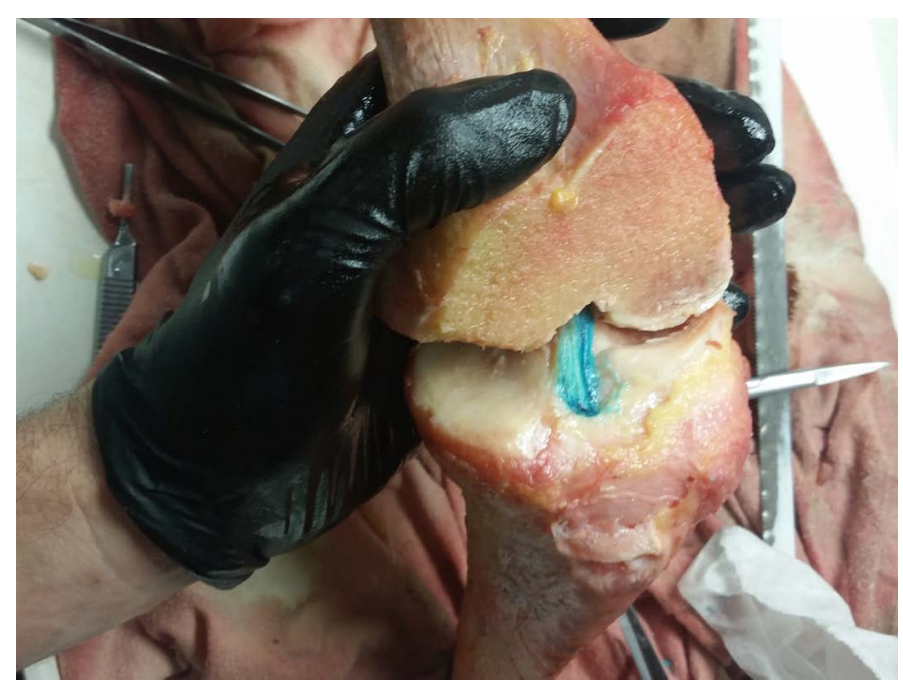

Figure 1. Exposed ACL fibers were stained with a blue dye mixture to reveal fiber bundles.

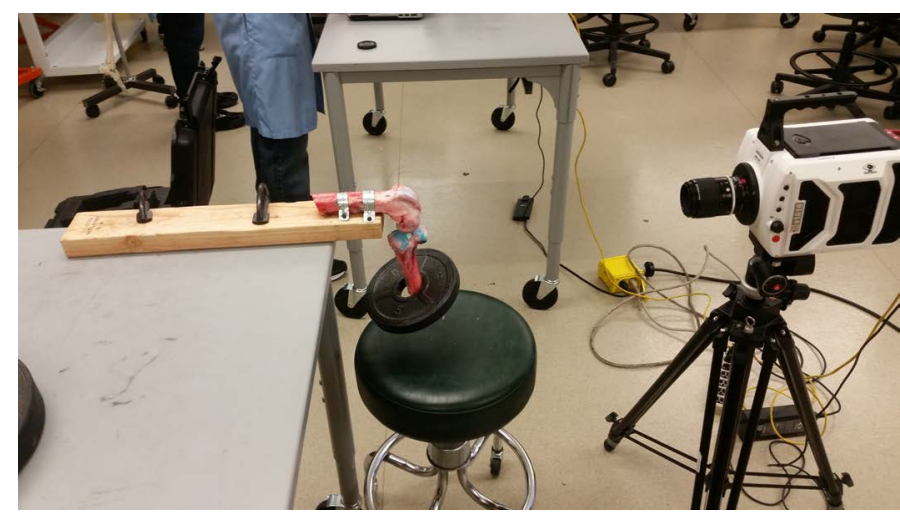

Figure 2. High-speed camera setup to videograph an ACL tear.

using video capture. Additionally, a valgus load was simultaneously placed on the knee joint, using manual manipulation, until the ACL completely failed. We cut the second ACL specimen from the medial side directly on the PL bundle and again placed a simultaneous valgus load until the ligament tore. To tear the third human ACL, a $9.5 \mathrm{~mm}$ hole was drilled at the distal end of the tibia, for a metal pin to be inserted. Next, we placed a $2.2 \mathrm{~kg}$ plate around the tibia above the 9.5 $\mathrm{mm}$ hole previously drilled; then the pin was inserted, with the weight resting on the pin. Appropriate lighting was used in a concentrated manner to illuminate the ACL architecture during video capture. With the weight in place, the ACL was cut $1-3 \mathrm{~mm}$ in depth to induce tearing of the fiber bundles. All three porcine ACL's were cut in the same manner as the third human ACL.

As the tearing took place, the high-speed camera (fastcam v1610, Tokyo, Japan) was focused on the ACL and filmed the unravelling bundles at a rate of 6,000 frames per second (fps). After the tear had taken place, the footage was then transferred to the computer program Phantom Camera Control Application (Tokyo, Japan) whereupon the researcher could more effectively view the fiber bundles as they unravel.

\section{Results}

\section{Human ACL}

All three-human model ACL's failed similarly as a result of the scalpel cut, but there were noticeable differences in comparison to the porcine model. Human ACL fiber bundles were tightly oriented together within the knee and there was not any visible physical separation of the AM and PL fiber bundles. The geometry of the fibers (from origin to insertion site) were structured in a cylindrical shape. The AM and PL fiber bundles were very similar in lengths and it was relatively easy to apply stress on either fiber bundle; flexion of the knee applied stress on the AM bundle, while extension applied stress on the PL bundle. Video analysis of the tears showed the AM bundle tightly wound around the PL bundle (see videos in supplementary material). Every video tear showed a small ripple going through the AM fiber immediately prior to failure. The ripple was initiated by a cut to the ACL and was viewed immediately before the ligament completely failed. In all three tears, the AM fiber bundle tore in an untwisting manner while the PL bundle acted as a post for the AM bundle. The PL fiber bundle then failed in a linear fashion. During the tearing process, both bundles tore in unison, one right after the other as if they were connected together. 


\section{Porcine ACL}

The porcine model displayed key features that differed relative to the human ACL model. Failure in the porcine model and the human model exposed three key species differences.

First, the porcine ACL was comprised of two fiber bundles that were separate from each other by a 1-2 mm gap and the geometry of the fibers were oriented in a triangular shape. The gap was apparent when the knee was extended as well as in a flexed position. A consequence of the bundle separation was that the porcine model ACL occupied a larger surface area at the insertion site on the tibia. Furthermore, the human ACL attached onto the tibia in a cylindrical structure, the porcine ACL fanned out and attached in a triangular structure.

In addition to this separation of the two bundles, it was apparent that the porcine PL bundle was shorter than the AM bundle. This became apparent in every flexed or extended position of the porcine model: The PL bundle became significantly more taunt, while the AM bundle stayed lax. The knee joint had to be manipulated in a specific position in order for the AM bundle to become taut, and even in this position, the AM bundle was not as stressed as the PL bundle. This directly affected the tearing dynamics of the two bundles. The AM bundle did not fail until the PL fiber bundle first failed, which then transferred the tension stress to the AM bundle resulting in its failure.

The final key difference was, due to their separation, that the two porcine ACL bundles tore independently of each other. When the AM bundle tear was induced with a cut, it would cease to tear, until the PL bundle tore first. This was because when the knee was in flexion or extension, the PL bundle, was the only bundle of the two that was taut because it was slightly shorter than the AM bundle.

A comparison of the human and porcine tears is shown in Figure 3.

\section{Significance}

Utilizing a high-speed camera to film the unravelling of the AM and PL bundles as the ACL failed provided insight into the structural morphology of the porcine model and human ACL structure. Understanding the intact structure of the human ACL and the failure mechanisms of the AM and PL fiber bundles may result in the engineering of better artificial or human allografts and in developing more effective repair and reconstruction methods. This could reduce or eliminate the many postoperative complications that accompany ACL reconstruction. Of particular interest in the human AM bundle tears was the ripple of a subpopulation of fibers after the tear was induced. It is likely that the compressive stress induced by the cut, before it tears the fibers, places a subpopulation of fibers under increased tension such that after they are cut, they release their energy as a ripple. However, the second human ACL tear was induced on the PL bundle, and yet the

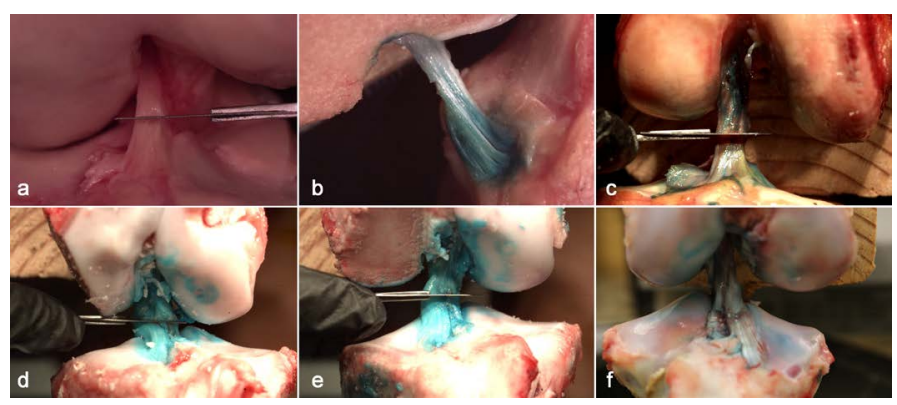

Figure 3. Still shots of high-speed videos exhibiting the artificially induced tear. Videos are available in the online, supplementary materials content. (a) human ACL 1, (b) human ACL 2, (c) human ACL 3, (d) porcine ACL 1, (b) porcine ACL 2, (c) porcine ACL 3.
AM bundle still exhibited a ripple on its anterior fibers when it failed. This suggests that the AM bundle may have subpopulations of fibers under varying tension. Prior research on the ACL has placed emphasis solely on the AM and PL bundles, but not on the subpopulation fibers themselves [4-7]. We did not observe a ripple in any other portion of the AM bundle or in the PL bundle but future work to make such observations may be important to understanding the microstructure of the AM bundle. Furthermore, we did not observe the same phenomenon on the porcine AM bundle, but that is likely due to its laxity in position of tearing.

The porcine model demonstrated key differences to the human model that may provide valuable insight to new methods by which the ACL could be reconstructed after it has undergone a tear. Specifically, the separation of the two fiber bundles resulted in the bundles tearing independently of each other. This may lead to increase stability within the porcine knee because the bundles are not dependent on the stability of the other. We are not aware of veterinary literature that characterizes porcine ACL tears. Additionally, the AM and PL fiber bundles are significantly separated at their insertion points, forming a triangular insertion into the tibia. We surmise that this results in stronger porcine ACL structure, since the greater surface area of the fiber bundles might lead to increased resistance to failure.

A human ACL repair or reconstruction that mimicked the porcine model, with two separate fiber bundles fanned out in a triangle insertion, may lead to greater knee stability and reduce the risk of retearing the ligament. More research needs to be done to find support for this hypothesis.

\section{Limitations}

This project focused on the filming of ACL tearing using a highspeed camera. We were able to visualize the unravelling of the ACL fiber bundles in both human and porcine models. We recognize that tensile and shear forces were not measured; future work will combine videography with traditional biomechanics methodologies. The knee was placed in a flexed position for convenience of its filming, but we acknowledge that 90 degrees of flexion is not typically when the ACL tears. We would also like to attempt filming while the knee is extended (or at least only slightly flexed). To do so, we will need to innovate a better filming window into the articular space. Furthermore, we will explore twisting the femur and tibia in opposite directions, pulling the proximal tibial head anteriorly, and placing a valgus force on the knee while in flexion; all stresses that the knee experiences when the ACL is torn. These future research efforts will enable a more accurate representation of the ACL within the motion of a natural tear without the introduction of a cut.

\section{Acknowledgements}

The authors wish to thank individuals who donated their bodies and tissues for the advancement of education and research. The authors also acknowledge the generosity of financial benefactors who contributed to the Brigham Young University Office of Research and Creative Activities (ORCA) Mentoring Environment Grant and Student Research Grant that funded this project.

\section{References}

1. Griffin LY, Agel J, Albohm MJ, Arendt EA, Dick RW, et al. (2000) Noncontact anterior cruciate ligament injuries: risk factors and prevention strategies. $J$ Am Acad Orthop Surg 8: 141-150.

2. Paterno MV, Rauh MJ, Schmitt LC, Ford KR, Hewett TE (2014) Incidence of Second ACL Injuries 2 Years After Primary ACL Reconstruction and Return to Sport. Am J Sports Med 42: 1567-1573. [Crossref] 
3. Amis AA (2012) The functions of the fibre bundles of the anterior cruciate ligament in anterior drawer, rotational laxity and the pivot shift. Knee Surg Sports Traumatol Arthrosc 20: 613-620. [Crossref]

4. Lorbach O, Pape D, Maas S, Zerbe T, Busch L, et al. (2010) Influence of the anteromedial and posterolateral bundles of the anterior cruciate ligament on external and internal tibiofemoral rotation. Am J Sports Med 38: 721-727. [Crossref]

5. Kennedy JC, Weinberg HW, Wilson AS (1974) The anatomy and function of the anterior cruciate ligament. As determined by clinical and morphological studies. $J$ Bone Joint Surg Am 56: 223-235. [Crossref]
6. Meyer EG, Haut RC (2008) Anterior cruciate ligament injury induced by internal tibial torsion or tibiofemoral compression. J Biomech 41: 3377-3383. [Crossref]

7. Petersen W1, Zantop T (2007) Anatomy of the anterior cruciate ligament with regard to its two bundles. Clin Orthop Relat Res 454: 35-47. [Crossref]

8. Proffen BL, McElfresh M, Fleming BC, Murray MM (2012) A comparative anatomica study of the human knee and six animal species. Knee. 19: 493-499. [Crossref]

9. Gillespie M, Jorgensen A, Wilkes D, Wisco JJ (2013) Staining technique for MicroScribe 3D digitizing of anterior cruciate ligament and calcaneal tendon. Clin Anat 26: 1036-62.

Copyright: $(02018$ Smith DT. This is an open-access article distributed under the terms of the Creative Commons Attribution License, which permits unrestricted use, distribution, and reproduction in any medium, provided the original author and source are credited. 